\title{
MyoD and the Regulation of Myogenesis by Helix-Loop-Helix Proteins
}

\author{
Stephen J. Tapscott and Harold Weintraub \\ Fred Hutchinson Cancer Research Center, Seattle, Washington 98104
}

The MyoD protein is sufficient to orchestrate the coordinated expression of most, if not all, of the skeletal myogenic program in cell types derived from all three germ layers of the embryo. As such the regulation of MyoD expression and protein activity creates a nodal point, or master switch, that integrates the genetic and environmental influences on a cell. The expression and activity of the MyoD protein is regulated, in part, by interaction with members of a large family of proteins related to MyoD by sequence homology, the helix-loop-helix (HLH) ${ }^{1}$ proteins. This review will focus on the regulation of skeletal myogenesis by MyoD and related myogenic HLH proteins.

\section{Expression of a Single Gene is Sufficient} to Activate Myogenesis

The notion that a small number of genes, or a single gene, was capable of orchestrating an entire program of differentiation was postulated by Holtzer and colleagues based on the kinetics of the inhibition of myogenesis and erythrogenesis by the thymidine analog 5-bromo-2'-deoxyuridine (BrdU) (1-3). The critical observation that a large percentage of cells of the mouse fibroblast line C3H10T1/2 (10T1/2 cells) could be stably converted to myoblasts by a brief treatment with the DNA demethylating agent 5 -azacytidine $(4,5)$ provided additional evidence that a single gene was sufficient to activate myogenesis. This was directly demonstrated by genomic transfection experiments in which transfection of DNA isolated from myoblasts could convert $10 \mathrm{~T} 1 / 2$ cells to muscle at a frequency consistent with the transfer of a single myogenic locus, whereas transfection with $10 \mathrm{~T} 1 / 2$ cell DNA failed to convert $10 \mathrm{~T} 1 / 2$ cells to muscle $(6,7)$.

Subtraction hybridization screening was then used to identify myoblast-specific transcripts. One of the cDNAs that was expressed only in skeletal muscle was capable of directly converting $10 \mathrm{~T} 1 / 2$ cells to myoblasts without the necessity for treatment with azacytidine (8). This gene, MyoD, met the requirement for sufficiency of a single locus to convert a cell to a myoblast. To date, four different, but related (see below) genes have been cloned from muscle cells: $\mathrm{MyoD}$, myogenin, myf-5, and MRF4/Herculin/Myf6 (8-14). Each is sufficient to activate the myogenic program when expressed in a nonmuscle cell line and together they comprise the MyoD family of myogenic regulatory genes. At present, while quantitative differ-

Received for publication 9 November 1990.

1. Abbreviations used in this paper: bHLH, basic helix-loop-helix; BrdU, 5-bromo-2'-deoxyuridine; HLH, helix-loop-helix, MCK, muscle creatine kinase.

J. Clin. Invest.

(c) The American Society for Clinical Investigation, Inc. 0021-9738/91/04/1133/06 \$2.00

Volume 87, April 1991, 1133-1138 ences have been demonstrated (15), it is unclear whether they have qualitatively different functions.

The question arises, however, as to whether fibroblast cell lines, such as $10 \mathrm{~T} 1 / 2$, are cryptically committed to myogenesis and the activity of MyoD and related genes is only permissive for the expression of the muscle phenotype. This is apparently not the case because muscle specific genes can be activated by forced expression of MyoD (using a strong viral promoter) in a large number of cell lines, including neuroblastoma, melanoma, hepatoma, and teratocarcinoma (16). In addition, primary cultures of retinal pigment epithelial cells, chondrocytes, and dermal fibroblasts can be converted to skeletal muscle cells that are morphologically and biochemically indistinguishable from normal muscle cells (17). To the extent that it has been analyzed, the endogenous differentiation program of these converted cells has been extinguished. This suggests not only that MyoD is sufficient to activate the myogenic program without the $a$ priori cooperation of other muscle-specific factors, but also that the myogenic regulatory genes are capable of interacting with and suppressing the tissue-specific regulatory factors of the other cell types tested, even though these cells would not ordinarily express the myogenic genes.

\section{MyoD is a Muscle-specific Transcription Factor}

In vitro gel shift assays have demonstrated that MyoD protein binds DNA that contains a core consensus sequence specified by CANNTG (18). Paired MyoD binding sites are essential parts of several characterized enhancers of muscle-specific genes (18-21). MyoD binds cooperatively to the paired sites in the muscle creatine kinase (MCK) enhancer and deletion analysis has shown that the $\mathrm{NH}_{2}$-terminal 50 amino acids are necessary for this cooperativity (19). In enhancers that contain two MyoD binding sites both are necessary for full enhancer activity, arguing for the functional importance of cooperative binding. In contrast, some enhancers, such as that for alpha-cardiac actin, contain a single identified MyoD binding site (22). In this case the adjacent CArG and SP1 binding sites can functionally substitute for the second MyoD binding site, because the enhancer activity is dependent on the presence of all three sites.

The predicted protein sequence of MyoD shares a region of homology with the other myogenic regulatory genes as well as with other tissue-specific and ubiquitous transcription factors. Mutational analysis of MyoD has shown that this region is both necessary and sufficient for biological activity and for sequence-specific DNA binding (23). This region has the potential to form two amphipathic alpha-helices separated by a nonhelical loop, referred to as the (HLH) motif (24). Many members of the HLH protein family, including the MyoD family of myogenic regulatory genes, have a region rich in basic amino acids that is immediately amino-terminal to the HLH domain, which we will refer to as basic helix-loop-helix (bHLH) proteins. Murre et al. (25) demonstrated that tissue- 
specific bHLH proteins form heterodimers with the protein products of a ubiquitously expressed bHLH gene, E2A. The E2A gene, by differential splicing, encodes for at least three different bHLH proteins, E12, E47, and ITF1 $(25,26)$. These three proteins bind to the kappa-E2 enhancer of the kappa light chain immunoglobulin gene with a specificity for the same core CANNTG motif as MyoD. MyoD and related myogenic bHLH proteins have a relatively weak binding affinity to the CANNTG sites in the MCK enhancer, whereas the heterodimer formed with an E2A protein binds with much higher affinity $(25,27,28)$. A detailed mutagenesis study of the MyoD and E2A proteins showed that the HLH domain mediates protein-protein interactions, whereas the basic region interacts with DNA $(28,29)$.

Four separate genes have been identified that code for related bHLH proteins that bind to the E-boxes of the immunoglobulin enhancers: TFE3 (30), ITF2 (26), TFEB (31), and E2A (24). All four genes are transcribed in both B cells and non-B cells. In this review we will focus on the E2A proteins because their role in myogenesis has been most extensively studied. It should be stressed, however, that these other members of the E-box binding subfamily of bHLH proteins may provide additional combinatorial complexity to the regulation of the myogenic bHLH proteins.

Fusion proteins utilizing the Gal 4 DNA binding domain (which can bind to $\mathrm{Gal} 4$ binding sites upstream of a reporter chloramphinicol acetyl transferase [CAT] gene) fused to portions of either MyoD (Weintraub, H., unpublished data), myf5 , or the E2A proteins $(26,32)$ have been used to demonstrate that these bHLH proteins have an activation domain separate from the bHLH region. In the case of MyoD and E-box binding proteins ITF1 and ITF2, the activation domain maps to the amino-terminal region, the same region in MyoD that participates in cooperative binding. For myf-5 both the amino-terminal and carboxyterminal domains share function as activation domains.

Analysis of the binding site preferences for MyoD and E2A proteins shows that each has a slightly different preferred halfsite, giving homo- and heterodimers slightly different preferred binding sequences (33). A simple combinatorial model, therefore, would use the availability of a dimerization partner to target a preferred binding site and the bound complex would position the activation domains to interact with proteins that mediate transcription. Mutations of the MyoD basic region, however, showed that, compared to wild-type MyoD, some mutants bind with similar or better affinity to the MyoD binding sites in the MCK enhancer but do not activate transcription (28), arguing that the $\mathrm{NH}_{2}$-terminal activation domain of MyoD is usually "hidden" from the transcriptional machinery and that the "exposure" of the activation region can be regulated independently of DNA binding. This is supported by experiments where the Ga14-MyoD fusion protein is used to activate a Gal-CAT reporter. Here the MyoD activation domain can be dramatically unmasked by deletion of other sub-regions of MyoD (Weintraub, H., unpublished data).

Competition between HLH Proteins Regulates Myogenesis Replicating myoblast cell lines express both myogenic bHLH proteins and the E2A proteins in their nucleus, yet they fail to activate the myogenic program. Whereas MyoD activates some myoblast-specific genes in the replicating cell, the genes charac- teristic of terminal differentiation are activated only after the removal of growth factors (8). One possible mechanism of negatively regulating the activity of the myogenic bHLH proteins is by decreasing the available pool of E2A proteins through dimerization with the HLH protein Id (34). This protein shares the HLH domain but lacks an adjacent basic region. It has been demonstrated in vitro that Id dimerizes with the E2A protein products with a relatively high affinity, using the HLH region as a dimerization motif. By competing for HLH dimerization regions Id can prevent the formation of MyoD:E2A protein oligomers. Because Id lacks a region equivalent to the DNA binding domain of the bHLH proteins, Id containing heterooligomers do not bind the core CANNTG sequence. In vivo it has been demonstrated that forced expression of Id protein will inhibit the activity of MyoD on a reporter gene and inhibit myogenic conversion of $\mathrm{C} 2$ myoblasts. Similar inhibition is attained by overexpressing a MyoD protein with a deletion of the basic region (28), supporting the interpretation that Id inhibits the activity of the myogenic HLH proteins by competing with them for dimerization with the E2A proteins. Like the E2A proteins, Id is expressed in a wide variety of cell types suggesting that a similar mechanism is used to modulate bHLH activity in other lineages. In many of these lineages, including muscle, when the cells are induced to differentiate the level of Id mRNA declines (34).

The in vitro DNA binding assays suggested heterodimerization of the MyoD family of myogenic bHLH proteins with an E2A protein may be crucial for functional activity. Furthermore, gel retardation assays using nuclear extracts made from myotubes demonstrated that protein complexes are present that can bind to the MCK enhancer (18), and that these complexes contain both an E2A protein and either MyoD or myogenin (Weintraub, H., and A. B. Lassar, unpublished data). To determine whether the heterodimer is necessary for transcriptional acitivity, an in vivo assay of transcriptional activation by hetero- or homooligomers was performed by overexpressing MyoD or E2A proteins in COS cells (Weintraub, H., and A. B. Lassar, unpublished data). In this case the level of expression is thought to be high enough to make the amount of endogenous E2A proteins rate limiting. Expression of either MyoD alone or an E2A protein alone did not significantly activate a reporter construct containing the multimerized MyoD binding sites as an enhancer, even though nuclear extracts demonstrated the presence of DNA binding homooligomers. However, MyoD and E2A proteins acted synergistically when transfected together. The activity correlated with the presence of MyoD:E2A heterooligomers in the nuclear extracts.

A second in vivo demonstration of the importance of E2A protein interaction with the myogenic bHLH proteins was accomplished by inhibiting E2A protein synthesis by expressing an antisense $E 12$ construct in 10T1/2 cells (Weintraub, $H$., and A. B. Lassar, unpublished data). The ability to convert clones of antisense E12 expressing 10T1/2 cells to muscle either by treatment with 5-azacytidine or with a MyoD expressing retrovirus directly correlated with the residual level of E2A protein, demonstrating a genetic interaction between the myogenic bHLH and the E2A gene.

\section{Cell Cycle}

Withdrawal from the cell cycle is part of the terminal muscle differentiation program that can be regulated by MyoD. Mu- 
tants of the MyoD protein have been used to demonstrate that the cell cycle effect of MyoD can be separated from the ability of MyoD to induce differentiation $(35,36)$. Mutations of the basic region (the DNA binding domain) do not interfere with the cell cycle arrest that is mediated by MyoD; whereas, an intact HLH region is required. This suggests that the MyoD HLH region interacts with proteins mediating cell cycle control independently of DNA binding. MyoD can cause $G 1$ arrest in a large number of tissue culture cell types, even those that will not differentiate into muscle; it will also arrest cells transformed by known viral oncogenes such as ras.

\section{Regulation of MyoD Expression}

Evidence exists that both positive and negative trans acting factors regulate the transcription of MyoD. Positive autoregulation has been demonstrated for MyoD, myogenin, Myf-5, and MRF4/herculin/Myf-6(12, 13, 37, 38). In addition to autoregulation, the expression of any one of the MyoD family.of bHLH genes can, to some extent, activate the expression of other members of the myogenic bHLH group. This complex positive autoregulation circuitry may serve both to amplify the level of transcription and possibly to maintain a commitment to the myogenic lineage.

MyoD expression is repressed by trans acting factors in primary fibroblasts. The MyoD gene is located on the short arm of human chromosome 11 (39). When chromosome 11 is transferred from primary human fibroblasts to $10 \mathrm{~T} 1 / 2$ cells or B78 melanoma cells, the human MyoD locus on chromosome 11 is activated (40), and, in 10T1/2 cells, autoactivation also leads to the turn-on of the mouse MyoD gene. In contrast, in heterokaryons and whole-cell hybrids between fibroblasts and 10T1/ 2 cells the MyoD locus is not activated until specific human chromosomes are lost, suggesting that an important regulatory mechanism is the suppression of MyoD transcription in trans mediated by loci on separate chromosomes, probably on chromosomes 4 and $8(40)$. When the transferred human chromosome 11 is lost, the endogenous mouse myogenic regulatory genes can maintain their own expression.

The MyoD gene contains a $\mathrm{CpG}$ island that is virtually devoid of methylation in normal tissues and primary cell cultures (41). However, in the establishment of cell lines this CpG island becomes heavily methylated and, presumably, the MyoD gene becomes inactivated by this cis acting mechanism. It is possible that this methylation is selected for because of the loss or inactivation of trans acting repressors of MyoD transcription. 10T1/2 cells seem not to contain such repressors because introduction of an unmethylated MyoD gene via chromosome 11 transfer leads to MyoD activation. In this regard it is interesting to speculate that the expression of $M y o D$, and subsequently other muscle genes, in some tumors or cell lines may reflect the loss or inactivation of myogenic repressor genes in a previously transformed cell rather than the transformation of a cell previously committed to the muscle lineage.

\section{Growth Factors, Oncogenes, and the Regulation of Myogenesis}

Myogenesis is known to be inhibited by a large number of factors including a variety of activated oncogenes, serum, TGF-Beta, FGF, TPA, butyrate, and BrdU. These factors could target any of the several identified steps necessary for myogenesis: the transcription and translation of myogenic
bHLH genes, the transport of the proteins to the nucleus, dimerization or oligomerization with other bHLH proteins, DNA binding, the unmasking of an activation domain, and the interaction of this domain with other, as yet unidentified, transcription factors. It becomes possible, therefore, to determine which of the growth factors and oncogenes that affect myogenesis interact with the HLH proteins and to map their activity relative to a specific step in HLH function.

One mechanism by which serum factors may block differentiation is by prohibiting the formation of bHLH heterodimers, for example by maintaining expression of the Id protein. Id expression is maintained in myoblasts by serum and in the absence of serum Id levels in myoblasts decline (34). It is not known whether Id is directly controlled by fos or if its regulatory regions contain a serum-responsive element. In vivo footprinting of the MCK enhancer shows that the MyoD binding sites are not occupied in the replicating MM14 myoblast, but that with induction of differentiation by low serum medium, the sites become filled (42). Because both MyoD and E2A proteins are present in the myoblast, there must be regulation of either the formation of a complex or its ability to bind DNA. One study has shown that MyoD:E2A complexes in nuclear extracts are first detectable after withdrawal of serum factors (43), consistent with the notion that one of the mechanisms by which serum factors inhibit differentiation is by preventing MyoD:E2A dimer formation, possibly by maintaining high levels of Id protein. In contrast, another study (Weintraub, H., and A. B. Lassar, unpublished data) using different extraction techniques, finds MyoD:E2A complexes in replicating cells despite the presence of Id protein, suggesting that other mechanisms of regulation may also be important.

In contrast to the effect of serum, TGF-beta blocks myogenesis in serum free medium $(43,44)$, where Id expression is turned off. Whereas its activity in part can be attributed to the decline in the levels of MyoD and myogenin, TGF-beta will block the activity of myogenin even when myogenin expression is maintained from a transfected expression vector (43, 44). In this latter case, nuclear extracts demonstrate the presence of myogenin:E2A complexes that bind the MCK enhancer sequences in gel retardation assays (43). This would suggest that TGF-beta is working through a mechanism distinct from preventing heterooligomer formation, either by preventing DNA binding in vivo or preventing the ability of the bound ocmplex to activate transcription. Activated ras and overexpression of fos also inhibit both the transcription of the myogenic bHLH genes and interfere with the activity of their proteins (45). The effect of these agents on transcription of MyoD could be a manifestation of the interference with MyoD protein function, because the MyoD family of bHLH genes use positive autoregulatory feedback to maintain high levels of expression. It is not yet known whether ras, fos, and TGF-beta mediate their effects at the same or different steps in the function of myogenic bHLH proteins. None of these "reagents" inhibits the ability of Gal-MyoD fusion proteins to activate a Gal-CAT reporter construct (Weintraub, H., and A. B. Lassar, unpublished data), suggesting that inhibition occurs at a step before transcriptional activation, perhaps DNA binding.

The thymidine analogue BrdU blocks myogenic differentiation when incorporated into the DNA of the replicating myoblast apparently without effecting the ability of the MyoD family of bHLH proteins to activate transcription of the muscle 
structural genes (46). Transcription of MyoD and myogenin is extinguished in BrdU-substituted cells, but forced expression of MyoD in a BrdU-substituted cell will activate both myogenin and the genes of terminal differentiation. The endogenous MyoD gene, however, remains insensitive to trans activation, indicating that BrdU is blocking at a level that is upstream, either in cis or trans, of the MyoD gene. The fact that the effect of $\mathrm{BrdU}$ on MyoD transcription is reversible provides evidence that the commitment to the muscle lineage is not just maintained by the positive autoregulatory circuit of the myogenic bHLH genes because these genes are turned off in the presence of BrdU. Instead, another element, again either in cis or trans, must provide a memory of the commitment to make muscle.

The myc proteins also contain a bHLH domain and $c-m y c$ binds to the core CANNTG binding sequence of the other bHLH proteins expressed in skeletal muscle (47). The $m y c$ proteins have not been shown to dimerize in vitro with the E2A proteins, any of the MyoD family of bHLH proteins, or with Id. $C$-myc is expressed in skeletal myoblasts and shows a decline in steady-state mRNA levels when myoblasts are induced to differentiate (48). Overexpression of $c$-myc can inhibit myogenesis (49). The mechanism by which MyoD and $c-m y c$ interact is not understood, but it is interesting to note that the two also play antagonistic roles in regulating cell cycle where the HLH domain of MyoD is thought to be functionally important (see above).

As more is learned about the regulation of MyoD activity it will be interesting to map the activity of the different steps of bHLH function in primary tumors and transformed cell lines. Most, if not all, rhabdomyosarcomas express MyoD mRNA and protein $(39,50,51)$, yet the MyoD protein is not sufficient to fully activate terminal differentiation in these tumors. Whereas loss of heterozygosity on the short arm of chromosome 11 characterizes embryonal rhabdomyosarcomas (50), the locus responsible is distinct from the MyoD locus (39) (which is also on the short arm of chromosome 11) and, presumably, represents an antioncogene of the muscle lineage. Forced expression of MyoD in rhabdomyosarcoma cells can activate the expression of some aspects of the terminal muscle differentiation but is insufficient to activate all of the muscle program or to rescue the transformed phenotype (52).

Similar examples of partial MyoD activity are also documented in nonmuscle cell lines. For example, MyoD will not activate the full MCK enhancer in CV1 cells (16) but will activate either the alpha-cardiac actin enhancer (22) or a construct containing multimerized MyoD binding sites (Weintraub, $H$., unpublished data). In some cells, such as CV1 and HepG2 cells, where MyoD will not activate the MCK enhancer, formation of heterokaryons with $10 \mathrm{~T} 1 / 2$ cells will lead to expression of the reporter construct $(16,53)$. Whereas the factors responsible for this complementation have not been identified, it is apparent that some immortalized cell lines, as well as rhabdomyosarcomas, repress the activity of the myogenic bHLH proteins. Understanding how this repression occurs in both muscle and nonmuscle lineages may lead to common mechanisms of cell transformation.

\section{Perspective}

The sufficiency of a single gene to activate the skeletal muscle program in many differentiated cell types indicates that the complex interplay of genetic information that occurs during development converges on the suppression or activation of the MyoD family of bHLH genes. As might be anticipated the activation of these genes and the activity of their proteins are regulated at multiple levels. It is likely that many of the same mechanisms that regulate the expression and activity of myogenic bHLH genes will apply to the regulation of cell differentiation in many other lineages. In Drosophila neurogenesis, for example, lineage-restricted transcripts of the bHLH genes of the achaete-scute complex positively interact with a generally expressed E2A homologue, daughterless, and negatively interact with extramacrochaete, an HLH gene similar to Id (54). The apparent ubiquity of Id and E2A expression, and the ability of MyoD overexpression to extinguish the endogenous program of several different cell types suggests that the regulation of differential gene expression by HLH proteins is a general mechanism. In this regard, it is important to note that the MyoD family of myogenic bHLH genes is expressed only in skeletal muscle, not in smooth or cardiac muscle. Because all three muscle types express many of the same muscle structural genes it is likely that a parallel network of regulatory genes exist. Whether these will also be bHLH genes remains to be seen. The pivotal role that MyoD and related HLH genes play in development is underscored by their conservation during evolution. Homologues of MyoD have been cloned from $X$. laevis, D. melanogaster, and C. elegans (55-59).

More immediate clinical implications exploit the use of the MyoD family of myogenic bHLH proteins to convert fibroblasts to myoblasts for the purpose of research, diagnosis, and treatment. It is possible to convert fibroblasts or amniotic cells to skeletal muscle for the purpose of studying a muscle-specific protein, such as dystrophin or acetylcholine receptor proteins. For researchers this means that a readily available source of primary human tissue-primary human fibroblasts-can be converted to skeletal myoblasts to study the molecular biology of muscle cells in both normal and abnormal genetic backgrounds. Diagnostically the conversion of amniotic cells to myoblasts may be a useful adjunct to DNA-based prenatal screening. For example, in the case of Duchenne's muscular dystrophy, point mutations in the dystrophin gene that cause either early termination or lead to rapid protein degradation may not be detected by conventional DNA-based screening techniques, but would be expected to show abnormal protein amount or size in a protein-based screening procedure. Another diagnostic use noted above is the classification of soft tissue tumors based on the expression of myogenic bHLH proteins. To date, no muscle disease has been linked to bHLH genes, but given the redundancy implied by their overlapping activities it remains possible that subtle manifestations of phenotype instability may result from a mutation in one of the myogenic bHLH genes. Finally, using MyoD-mediated conversion, a large supply of myoblasts can be generated from donor fibroblasts for the purpose of myoblast transplantation, either to supply new muscle mass for the treatment of muscular dystrophies or as a vehicle to produce genetically engineered bioactive molecules for the treatment of systemic disease.

\section{Acknowledgments}

S. J. Tapscott is a McDonnell Fellow in Molecular Medicine and Cancer Research and supported by an NINCDS CIDA; H. Weintraub 
is a Howard Hughes Investigator and supported by National Institutes of Health funding.

\section{References}

1. O'Neill, M. C., and F. E. Stockdale. 1974. 5-Bromodeoxyuridine inhibition of differentiational kinetics of inhibition and reversal in myoblasts. Dev. Biol. 37:117-132.

2. Weintraub, H., G. L. Campbell, and H. Holtzer. 1972. Identification of a developmental program using bromodeoxyuridine. J. Mol. Biol. 70:337-350.

3. Weintraub, H., G. L. Campbell, H. Holtzer. 1973. Differentiation in the presence of bromodeoxyuridine is "all-or-none". Nat. New Biol. 244:140-142.

4. Taylor, S. M., and P. A. Jones. 1979. Multiple new phenotypes induced in 10T1/2 cells treated with 5-azacytidine. Cell. 17:771-779.

5. Konieczny, S. F., and C. P. Emerson, Jr. 1984. 5-Azacytidine induction of stable mesodermal stem cell lineages from $10 \mathrm{~T} 1 / 2$ cell: evidence for regulatory genes controlling determination. Cell. 38:791-800.

6. Lassar, A. B., B. M. Patterson, and H. Weintraub. Transfection of a DNA locus that mediates the conversion of $10 \mathrm{~T} 1 / 2$ fibroblasts to myoblasts. Cell. 47:649-656.

7. Konieczny, S. F., A. S. Baldwin, and C. P. Emerson, Jr. Myogenic determination and differentiation in $10 \mathrm{~T} 1 / 2$ cell lineages: evidence for a simple genetic regulatory system. Mol. Cell. Biol. 29:21-34.

8. Davis, R. L., H. Weintraub, and A. B. Lassar. 1987. Expression of a single transfected cDNA converts fibroblasts to myoblasts. Cell. 51:987-1000.

9. Wright, W. E., D. A. Sassoon, and V. K. Lin. 1989. Myogenin, a factor regulating myogenesis, has a domain homologous to MyoD. Cell. 56:607-617.

10. Edmondson, D. G., and E. N. Olson. 1989. A gene with homology to the myc similarity region of MyoD1 is expressed during myogenesis and is sufficient to activate the muscle differentiation program. Genes Dev. 3:628-640.

11. Braun, T., G. Buschhausen-Denker, E. Bober, E. Tannich, and H. H Arnold. 1989. A novel human muscle factor related to but distinct from MyoD induces myogenic conversion in $10 \mathrm{Tl} / 2$ fibroblasts. EMBO (Eur. Mol. Biol. Organ.) J. 8:701-709.

12. Rhodes, S. J., and S. F. Konieczny. 1989. Identification of MRF4: a new member of the muscle regulatory factor gene family. Genes Dev. 3:2050-2061.

13. Miner, J. H., and B. Wold. 1990 . Herculin, a fourth member of the MyoD family of myogenic regulatory genes. Proc. Natl. Acad. Sci. USA. 87:1089-1093.

14. Braun, T., E. Bober, B. Winter, N. Rosenthal, and H. H. Arnold. 1990

Myf-6, a new member of the human gene family of myogenic determination factors: evidence for a gene cluster on chromosome 12. EMBO (Eur. Mol. Biol. Organ.) J. 9:821-831

15. Yutzey, K. E., S. J. Rhodes, and S. F. Konieczny. 1990. Differential tran activation associate with the muscle regulatory factors MyhoD1, myogenin, and MRF4. Mol. Cell. Biol. 10:3934-3944.

16. Weintraub, H., S. J. Tapscott, R. L. Davis, M. J. Thayer, M. K. Adam, A. B. Lassar, and A. D. Miller. 1989. Activation of muscle specific genes in pigment, nerve, fat, liver, and fibroblast cell lines by forced expression of MyoD. Proc. Natl. Acad. Sci. USA. 86:5434-5438.

17. Choi, J., M. L. Costa, C. S. Mermelstein, C. Chagas, S. Holtzer, and H. Holtzer. 1990. MyoD converts primary dermal fibroblasts, chondroblasts, smooth muscle and retinal pigment epithelial cells into striated, mononucleated myoblasts and multinucleated myotubes. Proc. Natl. Acad. Sci. USA. 87:79887992.

18. Lassar, A. B., J. N. Buskin, D. Lockshon, R. L. Davis, S. Apone, S. D. Hauschka, and H. Weintraub. 1989. MyoD is a sequence-specific DNA binding protein requiring a region of myc homology to bind to the muscle creatinine kinase enhancer. Cell. 58:823-831.

19. Weintraub, H., R. Davis, D. Lockshon, and A. Lassar. 1990. MyoD binds cooperatively to two sites in a target enhancer sequence: occupancy of two sites is required for activation. Proc. Natl. Acad. Sci. USA. 87:5623-5627.

20. Piette, J., J.-L. Bessereau, M. Huchet, and J.-P. Changeux. 1990. Two adjacent MyoDI binding sites regulate expression of the acetyl choline receptor alpha subunit. Nature (Lond.). 345:353-355.

21. Rosenthal, N., E. B. Berglund, B. M. Wentworth, M. Donoghue, B. Winter, E. Bober, T. Braun, and H. Arnold. 1990. A highly conserved enhancer downstream of the human MLC $1 / 3$ locus is a target for multiple myogenic determination factors. Nucleic Acids Res. 18:6239-6246.

22. Sartorelli, V., K. A. Webster, and L. Kedes. 1990. Muscle-specific expression of the cardiac alpha-actin gene requires MyoD1, CArG-box binding factor, and Spl. Genes Dev. 4:1811-1822.

23. Tapscott, S. J., R. L. Davis, M. J. Thayer, P.-F. Cheng, H. Weintraub, and A. B. Lassar. 1988. MyoD1: a nuclear phosphoprotein requiring a myc homology region to convert fibroblasts to myoblasts. Science (Wash. DC) 242:405-411.

24. Murre, C., P. S. McCaw, and D. Baltimore. 1989. A new DNA binding and dimerization motif in immunoglobulin enhancer binding, daughterless, MyoD, and myc proteins. Cell. 56:777-783.

25. Murre, C., P. S. McCaw, H. Vassin, M. Caudy, L. Y. Jan, Y. N. Jan, C. V.
Cabrera, J. N. Buskin, S. D. Hauschka, A. B. Lassar, H. Weintraub, and D. Baltimore. 1989. Interactions between heterologous helix-loop-helix proteins generate complexes that bind specifically to a common DNA sequence. Cell. 58:537544.

26. Henthorn, P., M. Kiledjian, and T. Kadesch. 1990. Two distinct transcription factors that bind the immunoglobulin enhancer uE5/kE2 motif. Science (Wash. DC). 247:467-470.

27. Brennan, T. J., and E. N. Olson. 1990. Myogenin resides in the nucleus and acquires high affinity for a conserved enhancer element on heterodimerization. Genes Dev. 4:582-595.

28. Davis, R. L., P.-F. Cheng, A. B. Lassar, and H. Weintraub. 1990. The MyoD DNA binding domain contains a recognition code for muscle-specific gene activation. Cell. 60:733-746.

29. Voronova, A., and D. Baltimore. 1990. Mutations that disrupt DNA binding and dimer formation in the $\mathrm{E47}$ helix-loop-helix protein map to distinct domains. Proc. Natl. Acad. Sci. USA 87:4722-4726.

30. Beckman, H., L.-K. Su, and T. Kadesch. 1990. TFE3: a helix-loop-helix protein that activates transcription through the immunoglobulin enhancer $\mathrm{uE3}$ motif. Genes Dev. 4:167-179.

31. Carr, C. S., and P. A. Sharp. 1990. A helix-loop-helix protein related to the immunoglobulin E box-binding proteins. Mol. Cell. Biol. 10:4384-4388.

32. Braun, T., B. Winter, E. Bober, and H. H. Arnold. 1990. Transcriptional activation domain of the muscle-specific gene-regulatory protein myf5. Nature (Lond.). 346:663-665.

33. Blackwell, T. K., and H. Weintraub. 1990. A new binding-site selection technique reveals differences and similarities between MyoD and E2A DNAbinding specificities. Science (Wash. DC). 250:1104.

34. Benezra, R., R. L. Davis, D. Lockshon, D. L. Turner, and H. Weintraub. 1990. The protein Id: a negative regulator of helix-loop-helix DNA binding proteins. Cell. 61:49-59.

35. Sorrentino, V., R. Pepperkok, R. L. Davis, W. Ansorge, and L. Phillipson. 1990. Cell proliferation inhibited by MyoDl independently of myogenic differentiation. Nature (Lond.). 345:813-815.

36. Crescenzi, M., T. P. Fleming, A. B. Lassar, H. Weintraub, and S. A Aaronson. 1990. MyoD induces growth arrest independent of differentiation in normal and transformed cells. Proc. Natl. Acad. Sci. USA. 87:8442-8446.

37. Thayer, M. J., S. J. Tapscott, R. L. Davis, W. E. Wright, A. B. Lassar, and H. Weintraub. 1989. Positive autoregularion of the myogenic determination gene MyoD1. Cell. 58:241-248.

38. Braun, T., E. Bober, G. Buschhausen-Denker, S. Kohtz, K. H. Grzeschik H. H. Arnold, and S. Kotz. 1989. Differential expression of myogenic determination genes in muscle cells: possible autoactivation by the Myf gene products. EMBO (Eur. Mol. Biol. Organ.) J. 8:3617-3625.

39. Scrable, H. J., D. K. Johnson, E. M. Rinchik, and W. K. Cavanee, 1990 Rhabdomyosarcoma-associated locus and MyoDl are syntenic but separate loci on the short arm of human chromosome 11. Proc. Natl. Acad. Sci. USA 87:2182-2186.

40. Thayer, M. J., and H. Weintraub. 1990. Activation and repression of myogenesis in somatic cell hybrids: evidence for trans-negative regulation of MyoD in primary fibroblasts. Cell. 63:23-32.

41. Jones, P. A., M. Wolkowicz, W. M. Rideout III, F. A. Gonzales, C. M Marziasz, G. A. Coetzee, and S. J. Tapscott. 1990. De novo methylation of the MyoD1 CpG island during the establishment of immortal cell lines. Proc. Natl. Acad. Sci. USA. 87:6117-6121.

42. Mueller, P. R., and B. Wold. 1990. In vivo footprinting of a muscle specific enhancer by ligation mediated PCR. Science (Wash. DC). 246:780-786.

43. Brennan, T. J., D. G. Edmonson, and E. N. Olson. 1991. TGF-beta represses the actions of myogenin through a medhanism independent of DNA binding. Proc. Natl. Acad. Sci. USA. In press.

44. Heino, J., and J. Massague. 1990. Cell adhesion to collagen and decreased myogenic gene expression implicated in the control of myogenesis by transforming growth factor beta. J. Biol. Chem. 265:10181-10184.

45. Lassar, A. B., M. J. Thayer, R. W. Overell, and H. Weintraub. 1989. Transformation by activated ras or fos prevents myogenesis by inhibiting expression of MyoD1. Cell. 58:659-667.

46. Tapscott, S. J., R. L. Davis, A. B. Lassar, and H. Weintraub. 1989. 5Bromo-2' deoxyuridine blocks myogenesis by extinguishing expression of MyoD1. Science (Wash. DC). 245:532-536.

47. Blackwell, K. T., L. Kretzner, E. M. Blackwood, R. N. Eisenman, and H. Weintraub. 1990 . Sequence-specific DNA binding by the c-Myc protein. Science (Wash. DC). 250:1149-1151.

48. Endo, T., and B. Nadal-Binard. 1986. Transcriptional and posttranscriptional control of c-myc during myogenesis: its mRNA remains inducible in differentiated cells and does not suppress the differentiated phenotype. Mol. Cell. Biol. 6:1412-1421.

49. La Rocca, S. A., M. Grossi, G. Falcone, S. Alema, and F. Tato 1989. Interaction with normal cells suppresses transformed phenotype of v-myc-trans formed quail muscle cells. Cell. 58:123-131. 
lar differential pathology of rhabdomyosarcoma. Genes, Chromosomes, and Cancer. 1:23-35.

51. Dias, P., D. M. Parham, D. N. Shapiro, B. L. Weber, and P. J. Houghton 1991. Myogenic regulatory protein (MyoD1) expression in childhood solid tumors: diagnostic utility in rhabdomyosarcoma. Am. J. Pathol. 137:1283-1291.

52. Hiti, A. L., E. Bogenmann, F. Gonzales, and P. A. Jones. 1989. Expression of the MyoD1 muscle determination gene defines differentiation capability but not tumorgenicity of human rhabdomyosarcomas. Mol. Cell. Biol. 9:4722-4730.

53. Schafer, B. W., B. T. Blakely, G. J. Darlington, and H. M. Blau. 1990 Effect of cell history on response to helix-loop-helix family of myogenic regulators. Nature (Lond.). 344:454-458.

54. Campos-Ortega, J. A., and E. Knust. 1990. Molecular analysis of a cellula decision during embryonic development of Drosophila melanogaster: epidermogenesis or neurogenesis. FEBS (Fed. Eur. Biochem. Soc.) Lett. 190:1-10.

55. Hopwood, N. D., A. Pluck, and J. B. Gurdon. 1990. MyoD expression in the forming somites is an early response to mesoderm induction in Xenopus embryos. EMBO (Eur. Mol. Biol. Organ.) J. 8:3409-3417.

56. Harvey, R. P. 1990. The Xenopus MyoD gene: an unlocalized mRNA predates lineage restricted expression in the early embryo. Development (Camb.). 108:669-680.

57. Scales, J. B., E. N. Olson, and M. Perry. 1990. Two distinct Xenopus genes with homology to MyoD1 are expressed before somite formation in early embryogenesis. Mol. Cell. Biol. 10:1516-1524.

58. Michelson, A. M., S. M. Abmayr, M. Bate, A. Martinez-Airias, and T. Maniatis. 1990. Expression of a MyoD family member prefigures muscle pattern in Drosophila embryos. Genes Dev. 4:2086-2097.

59. Krause, M., A. Fire, S. Harrison, J. Preiss, and H. Weintraub. 1990 CeMyoD accumulation defines the body wall muscle cell fate during $C$. elegans embryogenesis. Cell. 63:907-919. 